\title{
Emergence of multiple drug resistance Vibrio cholerae 01 in East Delhi
}

\author{
Shukla Das ${ }^{1}$, Shilpee Choudhry ${ }^{1}$, Rumpa Saha ${ }^{1}$, Vishnampettai G. Ramachandran ${ }^{1}$, Kamaldeep \\ Kaur $^{1}$, B.L. Sarkar ${ }^{2}$ \\ ${ }^{1}$ The University College of Medical Sciences and GTB Hospital, University of Delhi, Delhi, India \\ ${ }^{2}$ National Institute of Cholera and Enteric Diseases, Beliaghata, Kolkata, India
}

\begin{abstract}
Introduction: Considering the changing geographical and temporal occurrence of Vibrio cholerae, there is a continuing need to monitor the strain characteristics and antibiotic resistance patterns of this pathogen. The present study was conducted to document the changing biology of $V$. cholerae isolates in and around Delhi, India, and the development of antibiotic resistance.

Methodology: A total of 1,424 stool samples or rectal swabs from patients with acute secretory diarrhoea admitted to Guru Teg Bahadur Hospital, Delhi, between January 2007 and December 2009 were processed using standard bacteriological methods. Strains identified as $V$. cholerae were further subjected to serogrouping, phage typing and antimicrobial susceptibility testing. Minimum inhibitory concentration (MIC) of gentamicin and tetracycline was determined.

Results: $V$. cholerae was isolated in 242/1,424 (17.0\%) specimens. Of these, the majority were $V$. cholerae O1 serotype (98.3\%) and serovar Ogawa. The drugs to which $V$. cholerae $\mathrm{O} 1$ isolates showed high levels of resistance were nalidixic acid, furazolidone, and cotrimoxazole throughout the study period, whereas strains were usually susceptible to chloramphenicol and cefotaxime. In 2007, there was a sudden increase of resistance to gentamicin and tetracycline, followed by a slow reversal to previous levels in subsequent years. The phage typing pattern (Basu and Mukherjee scheme) showed a dominance of phage type 2 throughout the study period.

Conclusion: The importance of reporting all cases of $V$. cholerae, should be greatly emphasized, with the ultimate goal of understanding the constantly changing resistance patterns of this pathogen.
\end{abstract}

Key words: Vibrio cholerae; MDR; gentamicin; tetracycline; phage typing

J Infect Dev Ctries 2011; 5(4):294-298.

(Received 01 June 2010 - Accepted 28 December 2010)

Copyright (C) 2011 Das et al. This is an open-access article distributed under the Creative Commons Attribution License, which permits unrestricted use, distribution, and reproduction in any medium, provided the original work is properly cited.

\section{Introduction}

Vibrio cholerae has been recognised as one of the common causes of bacterial diarrhoea throughout the developing world. Epidemics of cholera caused by toxigenic $V$. cholerae $\mathrm{O} 1$ and $V$. cholerae $\mathrm{O} 139$ represent a major public health problem [1]. The dynamic of its transmission is complex. Two serotypes of $V$. cholerae O1, Ogawa and Inaba, have been demonstrated to interconvert, owing to an antigenic shift in the $\mathrm{O}$ antigen lipopolysaccharide [2]. At Guru Teg Bahadur (GTB) Hospital, except for a few scattered reports of $V$. cholerae O1 Inaba in 1998 and 1999, $V$. cholerae O1 Ogawa (80\%) was the predominant serovar until 2003. During 2004 and 2006 , the frequency of isolation of $V$. cholerae $\mathrm{O} 1$ Inaba strains steadily increased [3]. The changing pattern of the antibiogram has also raised concerns about the treatment of cholera with antibiotics. The emergence of multiple antibiotic-resistant (MAR) isolates of $V$. cholerae [4] has added a new dimension to the variability in pathogenicity and potential virulence in precipitating diarrhoeal illness. Considering the changing geographical and temporal pattern of $V$. cholerae, there is a continuing need to monitor the characteristics and antibiotic resistance patterns of this pathogen. This study was conducted to document the changing biology and antibiotic trends of $V$. cholerae isolates in and around Delhi.

\section{Methodology}

The study was conducted in the Department of Microbiology, University College of Medical Sciences and GTB Hospital, Delhi, India, between January 2007 and December 2009. The study group included patients five years of age or older presenting with rapid onset of watery diarrhoea and vomiting. A total of 1,424 stool samples or rectal swabs were processed in the Department of Microbiology using standard bacteriological methods [5-7]. Stool samples 
Table 1. Drug resistance profile of $V$. cholerae O1 (2007-2009)

\begin{tabular}{|l|l|l|l|}
\hline & $2007(\mathrm{n}=58)$ & $2008(\mathrm{n}=85)$ & $2009(\mathrm{n}=95)$ \\
\hline Antibiotics & No (\%) & No (\%) & No (\%) \\
\hline Chloramphenicol & $1(1.7)$ & $0(0)$ & $1(1.05)$ \\
\hline Cefotaxime & $5(8.6)$ & $0(0)$ & $0(0)$ \\
\hline Ciprofloxacin & $28(48.2)$ & $29(34.1)$ & $30(31.5)$ \\
\hline Gentamicin & $24(41.3)$ & $0(0)$ & $0(0)$ \\
\hline Cotrimoxazole & $53(91.3)$ & $73(85.8)$ & $85(89.4)$ \\
\hline Tetracycline & $34(58.6)$ & $3(3.5)$ & $4(4.2)$ \\
\hline Furazolidone & $58(100)$ & $85(100)$ & $95(100)$ \\
\hline Nalidixic acid & $58(100)$ & $85(100)$ & $95(100)$ \\
\hline
\end{tabular}

from each patient were streaked on bile salt agar (BSA) and MacConkey agar and enriched into alkaline peptone water (APW) with subsequent plating.

Serogrouping was performed by slide agglutination with O1 (Difco, Maryland, USA) and O139 (National Institute of Cholera and Enteric Disease NICED, Kolkata, India) antisera. Antimicrobial susceptibility of confirmed $V$. cholerae isolates was performed on Muellor Hinton agar (MHA) by Kirby Bauer disc diffusion technique [7]. Each isolate was tested against eight antibiotics: chloramphenicol $(30 \mu \mathrm{g})$, cefotaxime $(30 \mu \mathrm{g})$, ciprofloxacin $(5 \mu \mathrm{g})$, cotrimoxazole $(25 \mu \mathrm{g})$, furazolidone $(50 \mu \mathrm{g})$, gentamicin $(10 \mu \mathrm{g})$, nalidixic acid $(30 \mu \mathrm{g})$ and tetracycline $(30 \mu \mathrm{g})[8,9]$. Minimum inhibitory concentrations (MICs) of gentamicin and tetracycline were determined for all the gentamicinand tetracycline-resistant isolates respectively by broth dilution method per standard guidelines. For quality control, Escherichia coli ATCC 25922 was used as the standard strain for MIC interpretation [8].

Phage typing was done at the Vibrio Phage reference centre (NICED Kolkata, India), following the conventional Basu and Mukherjee's method and the new phage typing scheme $[10,11]$.

\section{Results}

$V$. cholerae was isolated in $242 / 1,424(17.0 \%)$ specimens. Of these, the majority were $V$. cholerae O1 serotype, accounting for 238/242 (98.3\%) isolates, followed by non-O1 non-O139 in $4 / 242$ $(1.7 \%)$ samples. $V$. cholerae O139 serotype was not isolated in the study period. Figure 1 shows the distribution of $V$. cholerae serotypes and serovars between 2007 and 2009.
$V$. cholerae O1 Ogawa $(80 \%)$ was the predominant isolate from 2007 onward and remained so until 2009. $V$. cholerae O1 Inaba $(6.1 \%)$ existed for a brief period and was replaced by $V$. cholerae $\mathrm{O} 1$ Ogawa in subsequent years. Few $V$. cholerae $\mathrm{O} 1$ Hikojima (3\%) were isolated during this transition period. In contrast, non-O1 non-O139 (3.7\%) isolation was fairly constant, occurring sporadically in small numbers (Figure 1).

The drugs to which $V$. cholerae $\mathrm{O} 1$ isolates showed high level resistance throughout the study period were nalidixic acid, furazolidone and cotrimoxazole, whereas strains were usually susceptible to chloramphenicol and cefotaxime. The resistance pattern of $V$. cholerae $\mathrm{O} 1$ to these drugs did not show any change since 2001 , as had been reported previously [3]. Until 2006, V. cholerae O1 was fully susceptible (100\%) to gentamicin and tetracycline. However, in 2007, there was sudden rise of resistance to these antibiotics (Table 1), followed by a slow reversion to previous levels in subsequent years. All gentamicin-tetracycline resistant strains had the same resistance patterns and were sensitive to chloramphenicol and cefotaxime alone, while resistant to ciprofloxacin, nalidixic acid, furazolidone and cotrimoxazole. $\mathrm{MIC}_{50}$ and $\mathrm{MIC}_{90}$ of $V$. cholerae for gentamicin were $16 \mu / \mathrm{ml}$ and $64 \mu \mathrm{g} / \mathrm{ml}$ respectively, while for tetracycline they were $8 \mu \mathrm{g} / \mathrm{ml}$ and $32 \mu \mathrm{g} / \mathrm{ml}$ respectively, as depicted in Table 2 .

All the Hikojima isolates were sensitive to the tested drugs except cotrimoxazole.

The phage typing pattern (Basu and Mukherjee scheme) of V.cholerae O1 isolates showed 100\% clustering of phage type 2 throughout the study period. With the new typing scheme, which was more discriminatory, the clusters were distributed among phage T 26 and T 27 (95\%). 
Figure 1. Distribution of serotypes and serovars of $V$. cholerae isolated from stool samples

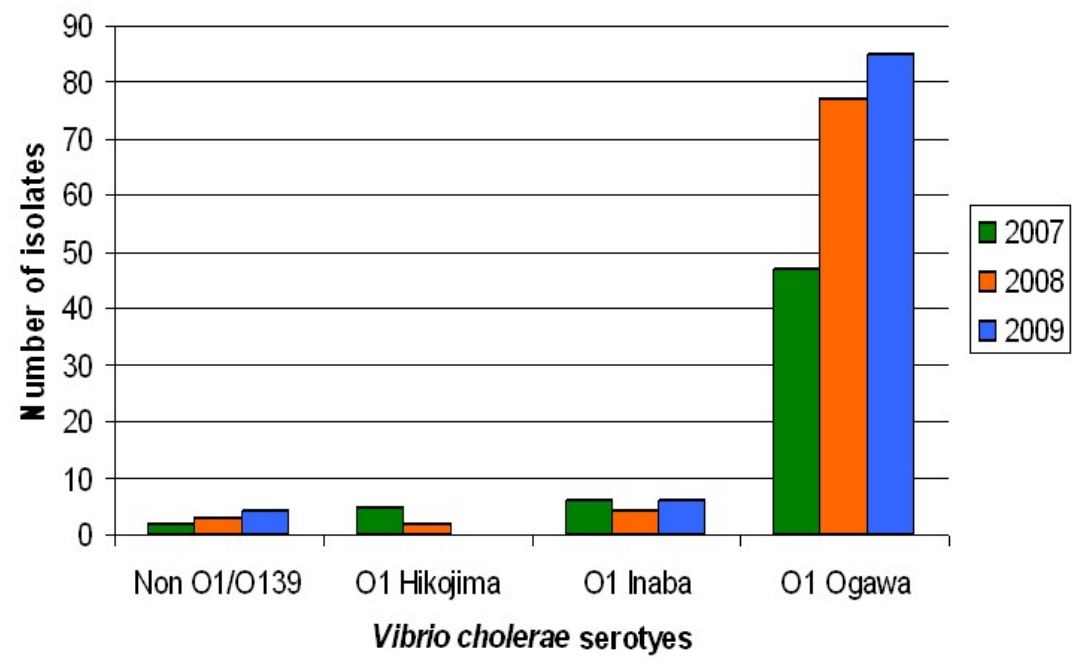

\section{Discussion}

Epidemic cholera spread from the Indian subcontinent to the rest of the world including America, Africa and Europe by 1950, though it ultimately remained in Asia. The ease and speed of travel, which has increased the number of travellers, contributed to the re-emergence of $V$. cholerae globally. Annual outbreaks of cholera are a regular occurrence in India. A high population density along with open drains and poor sanitation provide an optimal niche for the survival, sustenance and transmission of $V$. cholerae.

This study was an attempt to highlight the divergence of $V$. cholerae $\mathrm{O} 1$ isolates and the subsequent emergence of drug resistance during different time periods. The data demonstrate that the complexities of $V$. cholerae convertants, which predominate at different times with different strains, are presumably mediated via the host immune response.

At GTB Hospital, $V$. cholerae O1 Ogawa (80\%) was the predominant isolate until 2003 [3]. However, between 2004 and 2006, $V$. cholerae O1 Inaba (60\%) was isolated in large numbers [3] and, from 2007 onwards, $V$. cholerae $\mathrm{O} 1$ Ogawa (90\%) again became the predominant isolate and remained as such until 2009. This evident change to Inaba occurred after an interval of several years [3], which was followed by a re-emergence of Ogawa strains in subsequent years. Such unequal reciprocal interconversion perhaps occurs due to immune pressure in the population or antimicrobial selection pressure resulting in the mutation of the wbe (rfb) gene [2]. Thus serovar flux can be considered a strategy for the persistence and/or survival, and the temporality of such a flux is a reflection of the evolutionary pressure on the pathogen.

In India, cholera patients are treated with antibiotics along with a correction of dehydration status. Therefore, it becomes important to analyze the trends of antibiotic resistance among the clinical strains of $V$. cholerae. However, unlike other bacterial infections, little is reported about resistance patterns of $V$. cholerae, as laboratories do not routinely test susceptibility to different classes of antimicrobial agents. The emergence of multiple antibiotics resistant (MAR) $V$. cholerae with epidemic outbreaks has been reported in neighbouring Bangladesh $[4,12]$. The antibiotic resistance patterns of epidemic strains isolated from Bangladesh have documented reduced susceptibility to ampicillin, furazolidone, neomycin, streptomycin [13]. High-level resistance to choramphenicol, ampicillin, ciprofloxacin, neomycin, nalidixic acid and norfloxacin has been reported from Kolkata [14]. Outbreaks due to plasmid-mediated multiple drug resistant strains have also been associated with not only $V$. cholerae $\mathrm{O} 1$, but also with rough strain and non- $V$. cholerae O1. These strains demonstrated resistance to a spectrum of antibiotics, including chloramplenicol, tetracycline, ampicillin, sulphamethoxazole-trimethoprim, and streptomycin. Resistance determinants to aminoglycisides 
(kanamycin, gentamycin, neomycin) have also been reported in such strains $[15,16]$.

In the present study, V.cholerae $\mathrm{O} 1$ isolates showed $100 \%$ resistance against furazolidone, nalidixic acid and cotrimoxazole, suggesting that these drugs have a negligible role in the treatment of cholera. In contrast, most of the isolates were sensitive to chloramphenicol (98.3\%) and cefotaxime $(91.4 \%)$, which can be used as an alternative. We have reported previously about fluroquinolone resistance in children suffering from cholera in 2005 [17]. In subsequent years, ciprofloxacin resistance has largely remained between $30-40 \%$ [3]. All $V$. cholerae $\mathrm{O} 1$ isolates were sensitive to gentamicin and tetracycline in our area for the past several years. However, in 2007, 41.3\% and $58.6 \%$ of isolates developed resistance against gentamicin and tetracycline, respectively. The resistance to various antibiotics with a rapid shift, as reported in this study, is consistent with many reports indicating an enhanced mobility in genetic elements conferring resistance to $V$. cholerae strains. This makes it imperative that all isolates be constantly subjected to susceptibility testing and resistance patterns to each antibiotic be monitored.

Multiple drug resistance in $V$. cholerae can be attributed to either spontaneous mutation or to the horizontal transfer of resistance genes between members of gut coliform or other co-existing microflora and Vibrio [19]. The microcosm allows the existence of $V$. cholerae in non-culturable but viable conditions. During their human or animal passage, the sequence of events, which triggers a series of events and exchange of genetic material among the gut flora leading to rapid spread of drugresistant phenotypes both within and between bacterial species, remains to be defined $[15,19]$. Thus selection of such drug-resistant clones can lead to seasonal epidemics of cholera with the emergence of new clones replacing the existing clones.

Phage typing still remains a useful tool in the study of the epidemiology of cholera and is a recognized scheme that has been extensively developed and evaluated by the authors' reference laboratory, NICED, Kolkata, India. The results of phage typing were consistent with the overall countrywide epidemiological data, [20] which report type 27 to be the predominant type. The present findings corroborated previous studies [20,21].

In conclusion, a sudden increase in antibiotic resistance as reported here cannot be ignored; future epidemics in the MAR serotypes may be imminent.
The current study underscores the substantial mobility in the genetic element of $V$. cholerae and emphasises the need for continuous monitoring to ensure awareness of the changing traits of these capricious pathogens. The importance of reporting all cases should be emphasized, with the ultimate goal being a thorough understanding of the constantly changing and obscure epidemiology and resistance patterns of $V$. cholerae.

\section{References}

1. Janda JM, Powers C, Bryant RG, Abbott SL (1988) Current perspectives on the epidemiology and pathogenesis of clinically significant Vibrio spp. Clin Microbiol Rev 1: 245267.

2. Garg P, Nandy RK, Chaudhary P (2000) Emergence of Vibrio cholerae O1 biotype El Tor serotype Inaba from the prevailing $\mathrm{O} 1 \mathrm{Ogawa}$ serotype strains in India. J Clin Microbiol 38: 4249-4253.

3. Das S, Saha R, Kaur IR (2008) Trend of antibiotic resistance of Vibrio cholerae strains from East Delhi. Indian J Med Res 127: 478-482.

4. Shah M, Faruque M, Islam J, Ahmed QS, Biswas K, Faruque ASG, Nair GB, Sack RB, Sack DA, Mekalanos JJ (2006) An improved technique for isolation of environmental Vibrio cholerae with epidemic potential: monitoring the emergence of multiple antibiotic resistant epidemic strain in Bangladesh. J Infect Dis 193: 1029-1036.

5. Forbes BA, Sahm DF, Weissfeld AS (2002) Bailey \& Scott's diagnostic microbiology, $11^{\text {th }}$ edition. Missouri: Mosby Press 1069 p.

6. MacFaddin JF (1980) Biochemical tests for identification of medical bacteria. $2^{\text {nd }}$ edition. Baltimore: William and Wilkins Press 312 p.

7. World Health Organization (1993) Guidelines for cholera control. Geneva: World Health Organization.

8. CLSI 2008 Clinical and Laboratory Standard Institute Performance Standards for antimicrobial susceptibility testing $18^{\text {th }}$ Informational supplement: M100-S18 vol-28 No 1: $153 \mathrm{p}$.

9. Glass RI, Huq I, Alim A, Yunus M (1980) Emergence of multiple antibiotic resistance Vibrio cholerae in Bangladesh. J Infect Dis 142: 939-942.

10. Basu S, Mukerjee S (1970) A specific phage for pathogenic Vibrio cholerae, biotype El Tor (phage H74/64). Bull World Health Organ 43: 509-512.

11. Chattopadhyay DJ, Sarkar BL, Ansari MQ, Chakrabarti BK, Roy MK, Gosh AN, Pal SC (1993) New phage typing scheme for $V$. cholerae $\mathrm{O} 1$ biotype El Tor strains. J Clin Microbiol 31: 1579-1585.

12. Siddique A, Zaman K, Majumdar Y, Islam Q, Bashir I, Mutsuddy P, Eusof A (1989) Simultaneous outbreaks of contrasting drug resistant classical and El Tor $V$. cholerae O1 in Bangladesh. Lancet 2: 396.

13. Faruque SM, Saha MN, Asdulghani, Bag PK, Bhattacharya SK, Sach RB (2000) Genomic diversity among Vibrio cholerae 0139 strains isolated in Bangladesh and India between 1992 and 1998. FEMS Microbiol Lett 184: 279284.

14. Garg P, Chakraborty S, Basu I, Datta S (2000) Expanding multiple antibiotic resistance among clinical strains of 
Vibrio cholerare isolated from 1992-7 in Calcutta, India. Epidemiol Infect 124: 393-399.

15. Vakulenko SB, Mobashery S (2003) Versatility of aminoglycosides and prospects for their future. Clin Microbiol Rev 16: 430-450.

16. Sundaram SP, Murthy KV (1984) Transferable plasmid mediated drug resistance among non Vibro cholerae and rough strains of Vibrio cholerae from Tamil Nadu, India. J Hyg Camb 92: 59-65.

17. Das S, Goyal R, Ramachandran VGR, Gupta S (2005) Fluroquinolone resistance in Vibrio cholerae O1: emergence of EL Tor Inaba. Annal Trop Paed 25: 211-212.

18. Narang P, Mendiratta DK, Deotale VS, Narang R (2008) Changing patterns of Vibrio cholerae in Sevagram between 1990 and 2005. Ind J Med Microbiol 26: 40-44.

19. Speer BS, Shoemaker NB, Salyers AA (1992) Bacterial Resistance to tetracycline: mechanisms, transfer and clinical significance. Clin Microbiol Rev 5: 387-399.
20. Niyogi SK, De SP (1987) Prevalence of biotypes, serotypes \& phage types of Vibrio cholerae O1 in India (1975-1985). Indian J Med Res 85: 1-4.

21. Sarkar BL, Roy MK, Chakrabarti AK, Niyogi SK (1999) Distribution of phage type of Vibrio cholerae O1 biotype El Tor in Indian scenario (1991-98). Indian J Med Res 109: 204-207.

\section{Corresponding author}

Dr. Shilpee Choudhry

Department of Microbiology

UCMS \& GTB Hospital

Dilshad Garden, Delhi-110095

India

Phone: 9868234669

Fax:91-011-25095252

Email: drshilpee17@yahoo.in

Conflict of interests: No conflict of interests is declared. 